\title{
IMAGE RECONSTRUCTION FOR PET USING FUZZY POTENTIAL
}

\author{
Partha Pratim Mondal and K. Rajan \\ Department of Physics, Indian Institute of Science, Bangalore 560012, India \\ Phone: +9108022933280 \\ E-mail: partha(rajan)@physics.iisc.ernet.in
}

\begin{abstract}
Maximum likelihood (ML) and Maximum a-posteriori (MAP) are the most widely used algorithms for emission tomography (ET). MAP-approach heavily depends on Gibbs hyper-parameter for noise free reconstruction. Choosing a correct hyperparameter is difficult and time consuming task. Recently proposed median root prior (MRP) algorithm is a good alternative, but are prone to step like streaking effect. In this research work, a fuzzy logic based approach is proposed to overcome these shortcomings. Unlike traditional potential function, a fuzzy potential is used for modeling inter pixel interaction. Two basic operations viz. edge detection and fuzzy smoothing are performed sequentially during each iteration. The first operation is employed for the detection of edges (if present) in all the eight directions of a $3 \times 3$ neighborhood window. The second operation uses this edge information to perform fuzzy smoothing. Due to recursive nature of the reconstruction algorithm, both these operations are employed iteratively to reduce heavy noise produced due to dimensional instability [10], Simulated experimental results are obtained to show the feasibility of the proposed approach. These algorithms are also compared with other approaches such as, MAP and MRP by numerical measures and visual inspection. Algorithm evaluation shows promising results.
\end{abstract}

\section{INTRODUCTION}

In most of the medical imaging techniques, the data aquisition system ends up acquiring incomplete data. Incompleteness is due to the geometry of the imaging system and the nature of physical process involved. Penalized algorithms are found to be most efficient and reliable when dealing with incomplete data problem. A descriptive understanding of iterative image reconstruction algorithms for emission tomography (ET) can be found in [1][2]. Iterative algorithms offer good reconstruction at the cost of large computa- 
tional time. Currently, speed is not a central issue in ET. The computational complexity can be addressed to some extent by parallel computation [3] and by using fast processors (like, digital signal processor (DSP), media processor(MP) etc.) [4]. Many of the present day diagnostic imaging modalities like positron emission tomography (PET), single photon emission computed tomography (SPECT) demand good quality images. Though iterative algorithms like, maximum likelihood (ML) [1][2], maximum a-posteriori (MAP) [5][6][7] and median root prior (MRP) [8][9] algorithms are capable of generating good quality images but at the cost of artifacts like noise, oversmoothness, streaking effect [8] and hence require improvement.

Both ML and MAP are iterative data fitting problem, in which that estimate is chosen as the solution which makes the measurement data most likely. Regularization involves controlling the solutions (reconstructed images) that are desired. Regularization can be interpreted as a feedback process which demands continuous passage from image space to data space and vice-versa until pseudo-projections and measured projections match to the desired level of accuracy. The prior knowledge helps in producing the desired effect in the reconstructed image. For example, smoothing priors produce smooth reconstruction [5][6][7] whereas edge-preserving priors $[8][9]$ produce sharp reconstruction.

Fuzzy techniques have been successfully applied in image processing applications such as image restoration [11], morphology [12] etc.. In the present work we have extended fuzzy concepts to image reconstruction in PET. Prior distribution is defined by Gibbs distribution and the potential (which defines the nature of nearest neighbor interactions) is modeled using fuzzy rules.

\section{IMAGE RECONSTRUCTION ALGORITHMS FOR PET}

The measurements in PET $, y_{j}, j=1, \ldots, \mathrm{M}$ are modeled as independent Poisson random variables i.e, $y_{j} \sim \operatorname{Poisson}\left(\sum_{i=1}^{N} \lambda_{i} p_{i j}\right)$ for $j=1, \ldots, \mathrm{M}$, where $\lambda_{i}, i=1, \ldots, N$ are the mean parameters of the emission process and $p_{i j}$ is the probability that an annihilation in the $i^{\text {th }}$ pixel is detected in $j^{\text {th }}$ detector. The likelihood function i.e, the conditional probability for observing $\mathbf{Y}=\mathbf{y}$ given the emission parameter $\boldsymbol{\Lambda}=\lambda$ is the joint probability of the individual Poisson process i.e,

$$
P(y / \lambda)=\prod_{j=1}^{M}\left[\frac{\exp \left(-\sum_{i=1}^{N} \lambda_{i} p_{i j}\right)\left(\sum_{i=1}^{N} \lambda_{i} p_{i j}\right)^{y_{j}}}{y_{j} !}\right]
$$

Reconstruction algorithm proceeds by finding that estimate which maximizes the objective function i.e, ML or MAP depending upon the estimation technique employed. In ML, the objective function is taken as the likelihood function or equivalently log-likelihood function. Then, the task is the determination of that estimate $\lambda^{M L}$ which maximizes the objective function 
i.e,

$$
\lambda^{M L}=\max _{\lambda \geq 0}[\log P(y / \lambda)]
$$

The iterative equation solution for the above ill-posed reconstruction problem is first given by Shepp and Logan for PET [1]. The iterative update for generating $(k+1)^{t h}$ estimate from $k^{t h}$ estimate is,

$$
\lambda_{i}^{k+1}=\frac{\lambda_{i}^{k}}{\sum_{j=1}^{M} p_{i j}} \sum_{j=1}^{M} \frac{y_{j} p_{i j}}{\sum_{i=1}^{N} \lambda_{i}^{k} p_{i j}}
$$

On the other hand, MAP algorithm determines that estimate $\lambda^{M L}$ as the solution which maximizes the posterior density function $P(\lambda / y)$ or equivalently the $\log$ of $P(\lambda / y)$. Given a suitable prior $P(\lambda)$, MAP-reconstruction can be formulated as,

$$
\lambda^{M A P}=\max _{\lambda \geq 0}[\log P(y / \lambda)+\log P(\lambda)]
$$

Image field is assumed as Markov random field (MRF) [6] and by HammerseleyClifford theorem [13], image $\lambda$ is characterized by Gibbs distribution,

$$
P(\lambda)=\frac{1}{Z} e^{-\frac{2}{\beta} \sum_{i} \sum_{j \leftarrow N_{i}} w_{i j} V\left(\lambda_{i}, \lambda_{j}\right)}
$$

where, $\mathrm{Z}$ is the normalizing constant, for the distribution, $\beta$ is the Gibbs hyper-parameter, $w_{i j}$ is the weight of pixel $j \in N_{i}[5], N_{i}$ is the nearest neighbor set of pixcl $i$ and $V\left(\lambda_{i}, \lambda_{j}\right)$ is termed as the potential at site $i$ duc to the nearest neighbor elements $j$.

Solution for eqn.(4) is very difficult due to the complicated nature of prior. Green [5] has proposed one step late (OSL) approximation for an iterative update to the MAP-problem and is given by,

$$
\lambda_{i}^{k+1}=\frac{\lambda_{i}^{k}}{\left[\sum_{j=1}^{M} p_{i j}+\frac{1}{\beta} \sum_{j \in N_{i}}\left(\frac{\partial V\left(\lambda_{i}, \lambda_{j}\right)}{\partial \lambda_{i}}\right)_{\lambda_{i}=\lambda_{i}^{k}}\right]} \sum_{j=1}^{M} \frac{y_{j} p_{i j}}{\sum_{i=1}^{N} \lambda_{i}^{k} p_{i j}}
$$

Given the iterative OSL-algorithm (cqn.(6)), the next step is the proper modeling of the interacting potential $V\left(\lambda_{i}, \lambda_{j}\right)$ between the pixel at site $i$ and its neighbors $j \in N_{i}$. A large number of potentials (see [6][5][7]) have been suggested in the literature to produce desired image characteristics. It is a general nature of these potentials function to smooth the edges irrespective of the density class. The MAP reconstruction also involves prior knowledge of the hyperparameter values of the prior distribution, which are rarely available.

Recently, Alenius et. al. [8][9] has suggested median root prior algorithm for PET image reconstruction. The MRP formulation requires comparing of 
$\lambda_{i}$ with the median of the nearest neighbors i.e, $M_{b}=\operatorname{median}\left(\lambda_{j} ; j \epsilon N_{i}\right)$. More details can be found in [8][9]. The MRP-algorithm is given by,

$$
\lambda_{i}^{k+1}=\frac{\lambda_{i}^{k}}{\left[\sum_{j=1}^{M} p_{i j}+\frac{\left(\lambda_{i}^{k}-M_{b}\right)}{\beta M_{b}}\right]} \sum_{j=1}^{M} \frac{y_{j} p_{i j}}{\sum_{i=1}^{N} \lambda_{i}^{k} p_{i j}}
$$

The penalty term $\frac{\left(\lambda_{i}-M_{b}\right)}{\beta M_{b}}$ in (7) is piecewise linear with respect to the difference between the center pixel and local median. This difference can be non-zcro only if the image is not locally monotonic. Thus, noise is penalized because it is non-monotonic inside a small neighborhood. The local monotonic part represents the signal [9].

\section{FUZZY LOGIC BASED POTENTIAL FUNCTION}

Fuzzy rules provide a robust estimation for edge detection. A fuzzy logic based potential is proposed for edge-preserving reconstruction. This consists of two basic operations : fuzzy filtering and fuzzy smoothing. The proposed fuzzy technique is similar to that used by Ville et. al. for image restoration [11]. In the present work, the idea is expanded and adapted for PET.

\section{Fuzzy Derivatives for Edge Detection}

The derivative $\nabla^{k}(i, j)$ for pixel at $(i, j)$ along the direction $\hat{n}$ at $k^{t h}$ iteration is defined as,

$$
\nabla^{k}(i, j) \hat{n}=\left(\lambda^{k}(*, *)-\lambda^{k}(i, j)\right) \hat{n}
$$

where, $\lambda^{k}(*, *) \hat{n}$ represents the nearest pixel value along the unit directional vector $\hat{n}$. For example, the derivative along the west direction is defined as,

$$
\nabla^{k}(i, j) \hat{W}=\left(\lambda^{k}(i, j-1)-\lambda^{k}(i, j)\right) \hat{W}
$$

For identifying edge in a particular direction, three elementary derivatives are chosen (see fig.1). For example to detect an edge along $\hat{W}^{\gamma}$ direction, the derivatives are : $\nabla^{k}(i, j) \hat{W}, \nabla^{k}(i-1, j) \hat{W}$ and $\nabla^{k}(i+1, j) \hat{W}$ for a $3 \times 3$ window. It is safe to assume that if 2 out of 3 elementary derivatives are small, the edge is absent in the neighborhood. This is termed a $2: 3$ rule. For $\hat{n}=\hat{W}$ the fuzzy derivative is defined as follows :

If

$$
\left\{\begin{array}{l}
\nabla^{k}(i, j) \text { and } \nabla^{k}(i-1, j) \text { are small or } \\
\nabla^{k}(i, j) \text { and } \nabla^{k}(i+1, j) \text { are small or } \\
\nabla^{k}(i-1, j) \text { and } \nabla^{k}(i+1, j) \text { are small }
\end{array}\right.
$$

Then, $\nabla_{F}^{k}(i, j) \hat{W}$ is small

$$
\text { Else, } \nabla_{F}^{k}(i, j) \hat{W} \text { is large }
$$




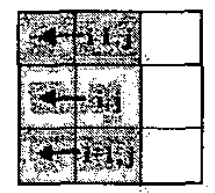

(a)

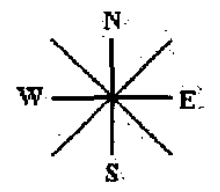

(b)

Figure 1: (a) $3 \times 3$ neighborhood of a central pixel $(i, j)$, showing the directional derivative along $\hat{W}$, (b) All the directions along which the the derivatives are taken.

Similarly, the values of the fuzzy derivatives $\nabla_{F}^{k}(i, j) \hat{n}$ for all the directions i.e, $\{\hat{W}, \hat{N}, \hat{S}, N \hat{W}, S \hat{W}, \hat{S E}, \hat{N E}\}$ are calculated.

\section{Membership Function}

Fuzzy sets are best represented by membership function. Membership function gives the degree of belongingness within the set. The membership function $\mu$ of a fuzzy set $\mathrm{X}$, maps the elements of $\mathrm{X}$ to the interval $[0,1]$ i.e, $X \stackrel{\mu}{\rightarrow}[0,1]$. To compute the value that expresses the degree to which the fuzzy derivative is small, we make use of fuzzy set small. Membership function for the property small is defined as,

$$
\mu^{k}(i, j) \hat{n}= \begin{cases}\text { small, } & \text { if } \nabla^{k}(i, j) \leq 0 \\ \text { large, } & \text { otherwise. }\end{cases}
$$

where, $k$ represents the iteration number and $\hat{n}$ represents the direction.

In iterative image reconstruction procedure, the estimate improves after each iteration and hence requires an updated membership function. Due to the iterative nature of the MAP algorithm, proposed membership function automatically updates the set small after each iteration. Similarly membership function is defined for all fuzzy derivatives $\nabla_{F}(i, j) \hat{n}$ along all the directions viz. $\{\hat{W}, \hat{N}, \hat{S}, N \hat{W}, \hat{S W}, \hat{S E}, \hat{N E}\}$.

\section{Fuzzy Penalization}

The next step is the penalization of pixels for which edges are not detected in the considered nearest neighborhood window, keeping others unaltered. The following rule is used for penalization :

If $\nabla_{F}^{k}(i, j) \hat{n}$ is small, Then $\Delta^{k}(i, j) \hat{n}=\nabla^{k}(i, j) \hat{n}$.

$$
\text { Else, } \Delta^{k}(i, j) \hat{n}=0
$$

where, $\Delta^{k}(i, j) \hat{n}$ is the feedback at site $(i, j)$ due to the adjacent pixel in the direction $\hat{n}$. Contributions are taken from all the eight directions. Hence, the total correction term $\Delta_{T}^{k}(i, j)$ for pixel at $(i, j)$ considering all the directions after $k^{\text {th }}$ iteration is given by,

$$
\Delta_{T}^{k}(i, j)=-\frac{1}{8} \sum_{\tilde{n}} \Delta^{k}(i, j) \hat{n}
$$


Replacing the error term $\sum_{j \in N_{i}}\left(\frac{\partial V\left(\lambda_{i}, \lambda_{j}\right)}{\partial \lambda_{i}}\right)_{\lambda_{i}=\lambda_{i}^{k}}$ in eqn.(6) by $\Delta_{T}^{k}(i)$, the OSL-algorithm modifies to,

$$
\lambda_{i^{\prime}}^{k+1}=\frac{\lambda_{i^{\prime}}^{k}}{\left[\sum_{j=1}^{M} p_{i^{\prime} j}+\frac{1}{\beta} \Delta_{r}^{k}\left(i^{\prime}\right)\right]} \sum_{j=1}^{M} \frac{y_{j} p_{i^{\prime} j}}{\sum_{o=1}^{N} \lambda_{o}^{k} p_{o j}}
$$

where, coordinates $(i, j)$ is denoted by a single coordinate $\left\{i^{\prime}=(i-1) *\right.$ $\sqrt{N}+j\}$. In the iterative image reconstruction procedure, the final correction term is fed back to update the pixel after each iteration. The iterations are continued until acceptable convergence is obtained.

\section{SIMULATED EXPERIMENTAL RESULTS}

\section{Simulated PET System}

The algorithm was tested on a simulated PET system. The PET system consists of a ring detector with 64 detectors and the object space is decomposed into $64 \times 64$ square pixels. The object space is a square region inscribed within the circle of detectors. Each element of the probability matrix $p_{i j}$ defines the probability of a photon getting detected in the detector $j$ after emanating from the object pixel $i$. For simplicity, we assumed that $p_{i j}$ depends only on the geometry of the measurement system. This is taken as the angle $\theta_{i j}$ seen by the center of the pixel $i$ into the detector tube $j[1]$ i.e, $p_{i j}=\frac{\theta_{i j}}{\pi}$. Before the reconstruction begins, the probability matrix $\mathbf{P}=\left[p_{i j}\right], i=1, \ldots, N$ and $j=1, \ldots, M$ is computed and stored.

For simulating measurement data, a Monte Carlo procedure is used in which each emission is simulated as follows [1][15]. First, a random pixel is chosen in the test phantom. The concentration of the radionuclei at the given pixel is assumed to be proportional to the emission density of the pixel. For each of the accepted emission point, a randomly oriented line (between 0 to $\pi$ radians) is selected and the pair of detectors this line intersects is found. The random line corresponds to the direction in which the pair of annihilated photons travel and the pixel corresponds to the arnihilation point in the phantom. The detectors with which this line intersects are assumed to detect this annihilation event and the count in the corresponding detector tube is incremented. In this way all the emissions are simulated and counted in the respective tubes. This is used as the measurement data for reconstruction. The mathematical phantom (also known as Shepp-Logan phantom) used in the present study is made up of nine elliptic objects having different sizes, orientations and density values. In fig.3, the image of the phantom is shown. We have used a source image with 100,000 counts. 

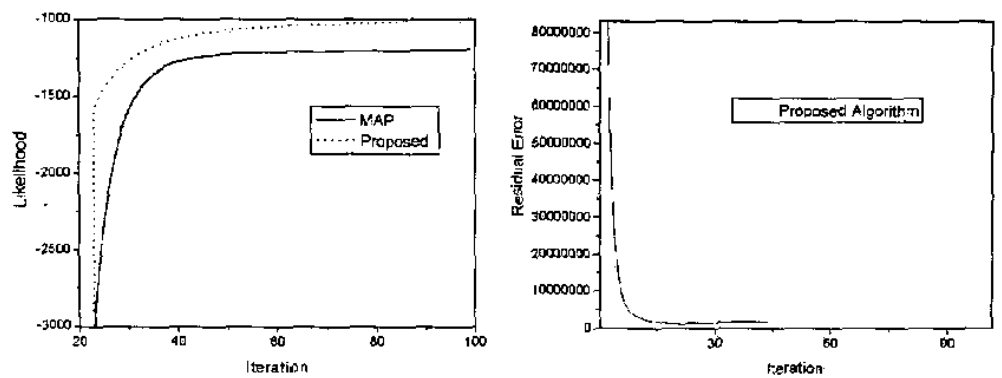

Figure 2: (a) Log-likelihood vs. iteration plot of the proposed algorithm, (b) Residual error vs. iteration plot of the proposed algorithm.

\section{Algorithm Evaluation}

All the evaluation tests defined in this section are carried out on a simulated PET system. The proposed algorithm is used on a $3 \times 3$ neighborhood window. The results arc compared with MAP and MRP algorithms. MAP with potential $V\left(\lambda_{i}-\lambda_{j}\right)=\sum_{j \in N_{i}}\left(\lambda_{i}=\lambda_{j}\right)^{2}$ with $\beta=2.5 \times 10^{4}$ is used in this study. That choice of $\beta$ is considered which gives the best estimate. The performances of the proposed new algorithm are evaluated using two different image-based quantitative criteria as given below :

Log-likelihood Test. The algorithms described in section II and III compute the estimate of the emission densities iteratively, hence log-likelihood function is an appropriate qualitative measure. For an estimate $\lambda^{k}$, the $\log$ likelihood function $l\left(\lambda^{k}\right)$ at $k^{t h}$ iteration is defined as,

$$
l\left(\lambda^{k}\right)=\sum_{j=1}^{M}\left[-\psi_{j}^{k}+y_{j} \log \psi_{j}^{k}-\log \left(y_{j} !\right)\right]
$$

where, $\psi_{j}^{k}=\sum_{i=1}^{N} \lambda_{i}^{k} P_{i j}$ is the pseudo-projection in the tube $j$.

The $\log$-likelihood values of the reconstructed images obtained using MAP and proposed algorithm are plotted against the iterations in fig.2(a). It is clearly evident that log-likelihood for the proposed algorithm converges faster compared to MAP-algorithm.

Residual Error. In iterative image reconstruction techniques, residual error is the most prefered evaluation test. This measures the deviation of the generated pseudo-projections $\psi_{j}^{k}$ of the reconstructed image from the observed projection data $y_{j}$. Residual error $\xi\left(\lambda^{k}\right)$ at $k^{t h}$-iteration is given by,

$$
\xi\left(\lambda^{k}\right)=\sum_{j=1}^{M}\left(y_{j}-\psi_{j}^{k}\right)^{2}
$$

where, $\psi_{j}^{k}=\sum_{i=1}^{N} \lambda_{i}^{k} P_{i j}$ is the pseudo-projection in the tube $j$. In fig.2(b), the residual errors of the reconstructed images using the proposed 


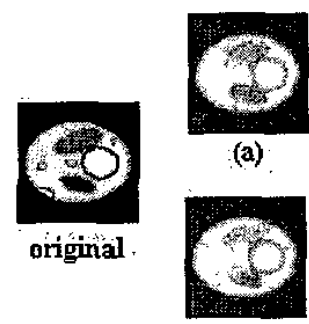

(d)

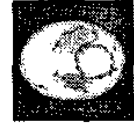

(b)

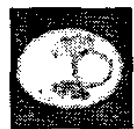

(e)

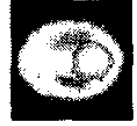

(c)

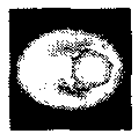

(i)

Figure 3: First row shows reconstruction after 50 iterations for (a) MAP, (b) MRP, (c) proposed algorithm. Second row shows reconstruction after 100 iterations for (d) MAP, (e) MRP, (f) proposed algorithm. For comparison, original test phantom is also shown.

algorithm is shown. This plots reveals the fact that residual error decreases with increase in iteration.

Visual Inspection. Final and the most important test is the visual inspection of the reconstructed images. In fig. $3,(\mathrm{a}, \mathrm{b}, \mathrm{c})$ and $(\mathrm{d}, \mathrm{e}, \mathrm{f})$ show the reconstructed images using MAP, MRP, proposed algorithm after 50 and 100 iterations respectively. For quality assessment, the original test image (phantom) is also shown. The images reconstructed using the proposed algorithm (fig.3(c),(f)) are more appealing and artifact free as compared to those reconstructed using MAP and MRP algorithms.

A small edge region of the reconstructed images using MAP, MRP and the proposed algorithm is shown in fig. 4 for further insight. MAP-reconstructed image shows over-smoothness (see fig.4(b)), while step like streaking effects are evident in MRP reconstructed image (see fig.4(c)) [9]. It can be seen that the images generated using the proposed algorithm are more appealing and free from streaking artifacts which is prominent in MRP-reconstructed images.

Line Plot. Line plots (see fig.5) show the image intensities along a prede-

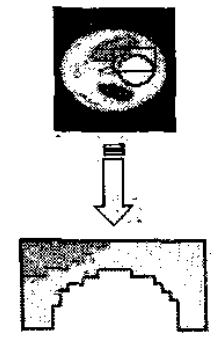

(a)
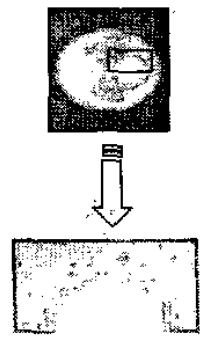

(b)

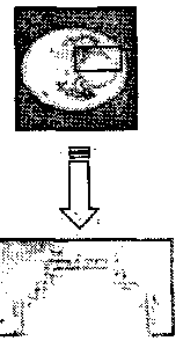

(c)

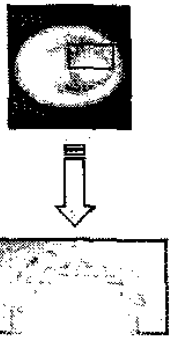

(d)

Figure 4: Zoomed version of the reconstruction after 100 iterations are shown: (a)

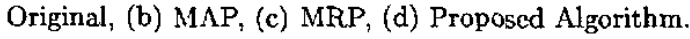



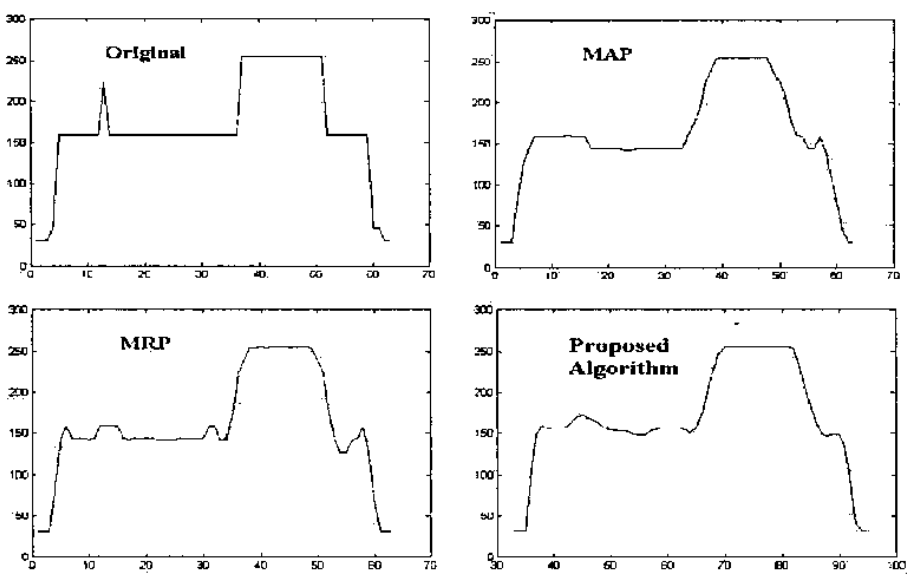

Figure 5: Line plots for the reconstructed images are shown after 80 iterations: (a) Original, (b) MAP, (c) MRP, (d) Proposed Algorithm.

fined line in the respective reconstructed images. The intensities are plotted along the central horizontal line through the reconstructed images. Line plots are shown for MAP, MRP and original test phantom for comparison. It is evident from these plots that MAP over smooths the reconstruction while MRP is capable of preserving the small details at the cost of streaking effect (see fig.5(c)) [9]. But the proposed algorithms generate reconstruction that are not too smooth and still preserves the edges.

\section{CONCLUSIONS}

A new approach is presented towards a better edge preserving reconstruction in PET. This is based on the application of fuzzy rule based techniques to model the pixel-pixel interacting potential in image lattice. Two basic fuzzy operations are performed: fuzzy filtering and fuzzy smoothing. These operations are performed iteratively to reduce heavy noise. Iterations are stopped when acceptable convergence is obtained. Simulated experimental PET studies reveal that the proposed algorithm is capable of producing estimates that are slightly better than the existing algorithms like MAP and MRP. There is no fear of convergence as the log-likelihood function is shown to converge with increasing iteration. Decrease in residual error confirms that the proposed algorithm is capable of producing estimates for which the generated pseudo-projections match closely to the measured projections and hence is the most likely estimate that has given rise to the observed projections. Visual representation of the reconstructed images using proposed algorithm is more appealing compared to MAP and MRP reconstructed images. The results are very encouraging. 


\section{REFERENCES}

[1] Y. Vardi, L. A. Shepp, and L. Kaufmann, "A statistical model for positron emission tomography", J. Amer. Stat. Assoc., vol. 80, pp. 8-37, 1985.

[2] L.A. Shepp and Y. Vardi., "Maximum likelihood estimation for emission tomography", IEEE Trans. on Med. Img. MI-1: pp. 113-121, 1982.

[3] C. M. Chen and S. Y. Lee, "Parallelization of the EM algorithm for 3-D PET image reconstruction", IEEE Trans. Med. Img., Vol.10, No.4, pp.513-522, Dec. 1991.

[4] K. Rajan, L. M. Patnaik and J. Ramakrishna, "High speed computation of the EM algorithm for PET inage reconstruction", IEEE Trans. Nucl. Sci., Vol. 41, No.5, pp.0-5, 1994.

[5] P.J. Green, "Bayesian reconstruction from emission tomography data using a modified EM algorithm", IEEE Trans. on Med. Img., vol.9, No.1, March, 1990.

[6] Z. Zhou, R. M. Leahy and J. Qi, "Approximate maximum likelihood hyperparameter estimation for Gibbs prior", IEEE Trans. on Img. proc., Vol.6, No.6, pp.844-861, June, 1997.

[7] J. Nuyts, D. Bequ, P. Dupont, and L. Mortelmans, "A Concave Prior Penalizing Relative Differences for Maximum-a-Posteriori Reconstruction in Emission Tomography", IEEE Trans. on Nuc. Sci., Vol. 49, No. 1, pp.56-60, Feb. 2002.

[8] S. Alenius and U. Ruotsalainen, "Using Local Median as the Location of Prior Distribution in Iterative Emission Tomography Reconstruction", IEEE Tran. Nucl. Sci., Vol.45, No.6, Dec. 1998.

[9] S. Alenius and U. Ruotsalainen, "Generalization of Median Root Prior Reconstruction", IEEE Trans. Med. Img., Vol.21, No.11, Nov., 2002.

[10] E. Veclerov and J. Llacer, "Stopping rule for MLE algorithm based on statistical hypothesis testing", IEEE Trans. on Med. Img., MI-6, pp. 313-319, 1987.

[11] D. V. Ville, M. Nachtegael, D. V. Weken, E. E. Kerre, W. Philips and I. Lemahieu, "Noise Reduction by Fuzzy Image Filtering" ,IEEE Trans. Fuzzy Sys., Vol. 11, NO. 4, Aug. 2003.

[12] M. Nachtegael and E. E. Kerre, "Decomposing and constructing fuzzy morphological operations over $\alpha$-cuts: Continuous and discrete case", IEEE Trans. Fuzzy Syst., vol. 8, pp. 615626, Oct. 2000.

[13] J. Besag, "Spatial interaction and the statistical analysis of lattice systems", Jl. of Royal Stat. Soc. B, Vol.36, pp. 192-236, 1974.

[14] S. Geman and D.Geman, "Stocastic relaxation, Gibbs distribution and the Bayesian restoration of images ", IEEE Trans. Pat. Anal. Mac. Intell., vol. PAMI-6, pp. 721-741, Nov., 1984.

[15] N. Rajeevan, K. Rajgopal and G. Krishna. "Vector-Extrapolated fast maximum likelihood estimation algorithms for emission tomography", IEEE Trans. on Med. Img., Vol. 11, No.1, March 1992.

[16] L. Kaufmann, "Implementing and accelerating the EM-algorithm for positron emission tomography", IEEE Trans. Med. Ing., Vol. MI-6, pp.37-51, Mar. 1987. 